\title{
Equações volumétricas para Eucaliptus sp no cerrado tocantinense
}

\author{
Thiêssa Tamilla de Carvalho Santana ${ }^{\mathrm{a}}$, Dayane Lucena Andrade ${ }^{\mathrm{a}}$, Maria Cristina Bueno Coelho ${ }^{\mathrm{a}^{*} \oplus \text {, }}$ \\ Mauro Luiz Erpen ${ }^{\mathrm{b}}$, José Fernando Pereira ${ }^{\mathrm{a}}$, Marcos Vinicius Cardoso Silva ${ }^{\mathrm{a}}$, \\ Mathaus Messias Coimbra Limeira ${ }^{\mathrm{a}}{ }^{\mathbb{D}}$, Maurilio Antônio Varavallo ${ }^{\mathrm{a}}$ \\ a Universidade Federal do Tocantins, Brasil \\ b Instituto Federal de Educação, Ciência e Tecnologia do Tocantins, Brasil \\ *Autor correspondente (mariacristina@uft.edu.br)
}

\section{N F O}

\section{Keyworks}

hard composting

forest inventory

eucalyptus

\begin{abstract}
A B S T R A C T
Volumetric equations for Eucaliptus sp in the cerrado tocantinense.

The objective of this work was to select volumetric equations for GG-100, VM-58 and seminal Eucalyptus clones, in Aliança, south of the state of Tocantins. Data were collected in a 7-year-old Eucalyptus plantation with a spacing of $2.5 \mathrm{~m} \times 4.0 \mathrm{~m}(1,000$ trees/ha). The selection of trees for cubage was carried out from a systematic forest inventory and stratified by clone and seminal for later determination of the diameter distribution. Cubing was performed using the Smaliam method on 48 trees with $\mathrm{DBH} \geq 12 \mathrm{~cm}$ until the entire diameter distribution was covered. 10 traditional mathematical models were adjusted for each species of eucalyptus. The best fitted models for each species were determined through the values of $\mathrm{R}^{2} \mathrm{aj}$ and $\mathrm{CV}(\%)$ together with the graphical analysis of residues. The best models were: lnv=$11,6833+1,4379 * \operatorname{lnd}+1,9246 * \operatorname{lnh}$ (Schumacher Hall) for VM-58 with the $\mathrm{R}^{2} \mathrm{aj}=0,88$ and CV\% $=5.00$, the $\operatorname{lnv}=$ model $6.6775+1,7840 *$ Ind (Husch) for GG-100 with $\mathrm{R}^{2} \mathrm{aj}=0.77$ and $\mathrm{CV} \%=34,89$ and $\operatorname{lnv}=-$ $9,6711+2,8759 * \operatorname{lnd}+0,0762 * \ln h$ (Schumacher Hall) for Seminal, with $\mathrm{R}^{2} \mathrm{aj}=0.91$ and $\mathrm{CV} \%=10,12$. Through the tukey test, no significant difference was observed in the DAP and volume variables between the clones.
\end{abstract}

\section{R E S U M O}

O objetivo do trabalho foi selecionar equações volumétricas para clones de GG-100, VM-58 e seminal de Eucalyptus, em Aliança, sul do estado do Tocantins. Os dados foram coletados em plantio de Eucalyptus, com 7 anos de idade com espaçamento de 2,5m x 4,0m (1.000 árvores/ha). A seleção das árvores para a cubagem foi realizada a partir de inventário florestal sistemático e estratificado por clone e seminal para posterior determinação da distribuição diamétrica. A cubagem foi feita pelo método de Smaliam em 48 árvores com DAP $\geq 12 \mathrm{~cm}$ até contemplar toda a distribuição diamétrica. Foram ajustados 10 modelos matemáticos tradicionais para cada espécie de eucaliptus. Os melhores modelos ajustados para cada espécie foram determinados através dos valores de $\mathrm{R}^{2}$ aj e CV (\%) juntamente com a análise gráfica de resíduos. Os melhores modelos foram: $\ln v=-11,6833+1,4379 * \operatorname{lnd}+1,9246 * \operatorname{lnh}$ (Schumacher Hall) para VM-58 tendo o $\mathrm{R}^{2} \mathrm{aj}=0,88$ e CV\% $=5,00$, o modelo $\ln v=6,6775+1,7840 * \operatorname{lnd}$ (Husch) para GG-100 com o $\mathrm{R}^{2} \mathrm{aj}=0,77$ e o $\mathrm{CV} \%=34,89$ e $\operatorname{lnv}=-9,6711+2,8759 * \ln d+0,0762 * \operatorname{lnh}$ (Schumacher Hall) para Seminal, com o $\mathrm{R}^{2} \mathrm{aj}=0,91$ e o $\mathrm{CV} \%=10,12$. Através do teste tukey, não foi observada diferença significativa nas variáveis DAP e volume entre os clones. 


\section{INTRODUÇÃO}

Para a produção de madeira, o gênero Eucalyptus compõe o grupo das principais espécies arbóreas utilizadas comercialmente no Brasil, sendo que em 2016, a área ocupada por plantios de Eucalyptus no país totalizou aproximadamente 5,70 milhões de hectares (IBÁ, 2017). No setor florestal brasileiro o Eucalyptus é um gênero de suma importância na produção de madeira, é amplamente utilizado nos florestamentos para aproveitamento como fonte energética, biomassa, celulose e papel, móveis, construção civil e madeira serrada, com destaque na utilização de multiprodutos florestais (IBÁ, 2017).

O gênero Eucalyptus é muito importante por apresentar alta produtividade e adaptabilidade nas mais diversas condições edafoclimáticas, observando-se que atualmente, as plantações de Eucalyptus estão presentes nas mais diversas regiões do mundo, em diferentes altitudes, tipos de solo e regimes pluviométricos (Vital, 2007).

O volume compõe uma das variáveis mais importantes para avaliar o potencial disponível do estoque de madeira e o potencial produtivo das florestas (Thomas et al., 2006). As equações volumétricas são bastante aplicadas nas estimativas e prognoses da produção madeireira, cujos parâmetros são definidos por regressão, instituindo um mecanismo eficaz para a quantificação da produção volumétrica de um povoamento florestal (Santana e Encinas, 2004).

A determinação do volume de árvores é, na maioria dos casos, o principal objetivo dos levantamentos florestais quando se trata de povoamentos designados a fins comercias. Portanto, o emprego de métodos de cubagem adequados, que garantam melhor precisão na determinação do volume individual de árvores, diminuirão o tempo e custo de trabalho (Carvalho et al., 2014). A altura e o diâmetro a altura do peito (DAP), são os dois principais parâmetros que são utilizados para o cálculo da área basal e do volume que existe em uma floresta (Viera et al., 2014).

$\mathrm{O}$ uso de fator de forma, equações volumétricas e modelos de afilamento são os procedimentos mais utilizados para estimar volume comercial (Machado et al., 2005). O método mais simples para determinar o volume real ou comercial de uma árvore é através do fator de forma (Silva e Paula, 1979). Diante disso, o objetivo do trabalho foi selecionar equações volumétricas para estimar a capacidade volumétrica dos clones de GG-100, VM-58 e seminal, na região sul do estado do Tocantins.

\section{MATERIAL E MÉTODOS}

O presente estudo foi realizado em um plantio comercial de Eucalyptus spp.com 7 anos de idade, e pertencente à empresa Projecto LTDA, localizada a $280 \mathrm{~m}$ de altitude e coordenadas geográficas $11^{\circ} 21^{\prime} 43^{\prime \prime}$ sul, e longitude $49^{\circ} 03^{\prime} 37^{\prime}$ " oeste, no município de Aliança do Tocantins, região Sul do estado do Tocantins, distante $167 \mathrm{~km}$ da capital Palmas.

O clima, de acordo com Köppen, é do tipo AWtropical de verão úmido e período de estocagem no inverno, precipitação anual tem variação entre 1500 a $2100 \mathrm{~mm}$. A caracterização clima segundo Thornthwaite e Mather é C2wA'a", clima úmido sub úmido com moderada deficiência hídrica no inverno, evapotranspiração potencial média anual de $1.500 \mathrm{~mm}$, distribuindo-se no verão em torno de $420 \mathrm{~mm}$ ao longo dos três meses consecutivos com temperatura mais elevada. A temperatura média anual situa-se entre $25^{\circ} \mathrm{C}$ e $26^{\circ} \mathrm{C}$. O solo predominante na área de estudo é classificado como Latossolo Vermelho-Amarelo (SEPLAN, 2012).

A área do plantio corresponde a 109 hectares os quais se subdividem em três clones Eucalyptus urophylla $x$ Eucalyptus camaldulensis (VM 58), 37 ha, Eucalyptus urophylla $x$ Eucalyptus grandis (GG100), 48 ha e um plantio seminal de sementes híbridas de Eucalyptus. urophylla $x$ Eucalyptus grandis, 24 ha, plantados no espaçamento 2,5 x 4,0 $\mathrm{m}$ totalizando a média de 1000 árvores por hectare.

$\mathrm{O}$ banco de dados foi determinado a partir de inventário florestal sistemático estratificado por clone, realizado em 2018 com 42 parcelas permanentes de $800 \mathrm{~m}^{2}$ (80 árvores por parcela). Foi medido o DAP de todas as árvores das parcelas e a altura total foi mensurada somente em $10 \%$ dos indivíduos. A altura foi medida com um uso de um clinômetro. A partir destes dados, os diâmetros foram distribuídos em intervalos de classes de 2 $\mathrm{cm}$, com a finalidade de determinar o número de indivíduos a serem cubados para estimativa do volume real para o povoamento florestal.

A seleção das árvores para a cubagem foi realizada a partir da distribuição da frequência diamétrica. Foi feita a seleção e a cubagem rigorosa de árvores-amostra abatidas com DAP $\geq 12 \mathrm{~cm}$ de forma a contemplar toda a distribuição diamétrica. $\mathrm{O}$ número de árvores a serem cubadas foi definido através da fórmula (equação 1) descrita por Mello (2004):

$$
n=\frac{t^{2} \cdot(C V \%)^{2}}{E^{2} \%}
$$

Em que: $\mathrm{t}=$ valor tabelado (Student) conforme o "n" e o nível de precisão requerido de $95 \%$ de probabilidade de confiança; $\mathrm{CV} \%=$ coeficiente de 
variação em percentagem; $\mathrm{E}=$ erro pré-estabelecido, neste caso utilizado $10 \%, \mathrm{n}=$ número de árvores a serem cubadas rigorosamente.

Foi necessário estabelecer uma amostra piloto, para definir quantas árvores deveriam ser cubadas rigorosamente. Essa amostra resultou na cubagem de 48 árvores, desse modo tem-se a ideia da variabilidade entre os volumes das árvores que compõem a população considerada (Mello, 2004).

Seguindo este preceito foram cubadas 48 árvores-amostra para determinação do volume rigoroso utilizando a metodologia de Smalian (equação 2), onde mostra que o volume de cada seção foi somado para obter o volume total das toras conforme a expressão (Finger, 1992):

$v=v_{0}+\sum_{i=1}^{n} v_{i}+v_{c}$

Em que: $v=$ volume, $v_{0}=$ volume do toco, $v_{i}=$ volume das secções transversais e $v_{c}=$ volume do cone.

Com os dados obtidos na cubagem foram calculados os volumes individuais para cada uma das árvores-amostra. $\mathrm{O}$ fator de forma artificial (equação 3) foi obtido pela razão entre o volume rigoroso e o volume do cilindro com diâmetro 1,3 m de acordo com a expressão (Finger, 1992):

$$
f_{1,30}=\frac{\text { volume rigoroso }}{\text { volume do cilindro com diametro } d_{1,3}}
$$

Em que: $f_{1,30}=$ fator de forma artificial; $d_{1,3}=$ diâmetro medido a $1,30 \mathrm{~m}$ da altura total da árvore.

Os dados destas árvores serviram de base para o ajuste dos modelos de volume relacionados na Tabela 01 . Os modelos de volume testados foram selecionados na literatura existente sobre o tema.

Tabela 1: Modelos matemáticos para determinação do volume individual em $\mathrm{m}^{3}$ do plantio comercial de Eucalyptus em Aliança-TO.

\begin{tabular}{lcl}
\hline $\mathrm{N}^{\circ}$ & Função & \multicolumn{1}{c}{ Modelo } \\
\hline 1 & Hohenald-Krenn & $v=\beta_{0}+\beta_{1} \cdot d+\beta_{2} \cdot \mathrm{d}^{2}$ \\
2 & Stoate & $v=\beta_{0}+\beta_{1} \cdot d^{2}+\beta_{2} \cdot\left(d^{2} \cdot \mathrm{h}\right)+\beta_{3} \cdot \mathrm{h}$ \\
3 & Näslund & $v=\beta_{1} \cdot d^{2}+\beta_{2} \cdot\left(d^{2} \cdot \mathrm{h}\right)+\beta_{3} \cdot\left(d \cdot \mathrm{h}^{2}\right)+\beta_{4} \cdot \mathrm{h}^{2}$ \\
4 & Meyer & $v=\beta_{0}+\beta_{1} \cdot d+\beta_{2} \cdot d^{2}+\beta_{3} \cdot(d \cdot \mathrm{h})+\beta_{4} \cdot\left(d^{2} \cdot \mathrm{h}\right)$ \\
5 & Meyer (modificado) & $v=\beta_{0}+\beta_{1} \cdot d+\beta_{2} \cdot d^{2}+\beta_{3} \cdot(d \cdot \mathrm{h})+\beta_{4} \cdot\left(d^{2} \cdot \mathrm{h}\right)+\beta_{5} \cdot \mathrm{h}$ \\
6 & Schumacher-Hall & $\ln v=\beta_{0}+\beta_{1} \cdot \ln d+\beta_{2} \cdot \operatorname{lnh}$ \\
7 & Spurr logaritimico & $\ln v=\beta_{0}+\beta_{1} \cdot \ln \left(\mathrm{d}^{2} \mathrm{~h}\right)$ \\
8 & Husch & $\ln v=\beta_{0}+\beta_{1} \cdot \ln d$ \\
9 & Brenac & $\ln v=\beta_{0}+\beta_{1} \cdot \ln d+\beta_{2} \cdot\left(\frac{1}{d}\right)$ \\
& & $v=\beta_{0}+\beta_{1} \cdot \ln \left(\mathrm{d}^{2} \mathrm{~h}\right)$
\end{tabular}

Em que: $\mathrm{v}=$ volume individual $\mathrm{em} \mathrm{m}^{3} ; \mathrm{h}=$ altura total $(\mathrm{m}) \mathrm{e} \mathrm{d}=$ diâmetro a altura do peito em $\mathrm{cm} ; \beta_{0}, \beta_{1}, \beta_{2}, \beta_{3}, \beta_{4} e \beta_{5}=$ coeficientes da regressão.

\section{Critérios para seleção do modelo volumétrico}

Para seleção do modelo matemático que melhor descreve a estimativa do volume, foram avaliados o erro padrão residual (Syx\%), os coeficientes de determinação $\left(\mathrm{R}_{\text {aj }}^{2}\right)$, a dispersão gráfica dos resíduos e o valor $\mathrm{F}$. $\mathrm{O}$ erro padrão da estimativa em porcentagem informa a qualidade do ajuste e o quanto, relativamente, o modelo erra em média ao estimar a variável dependente. Quando a variável dependente sofreu algum tipo de transformação, é necessário que se faça o recálculo do $S y \mathrm{x}$ (equação 4), para que esse possa ser comparável aos das equações cuja variável dependente não sofreu transformação. Conforme utilizados por Batista et al. (2001), essa correlação é efetuada através da seguinte fórmula:

$$
\begin{aligned}
& \text { Syx }=\sqrt{\frac{\sum_{i=1}^{n}\left(h_{i}-h i\right)^{2}}{n-p}} \\
& S y x \%=\left(\frac{S y x}{\bar{h}}\right) * 100
\end{aligned}
$$

Em que: $h_{i}=$ Altura observada; $h \hat{\imath}=$ Altura estimada pela equação; $\bar{h}=$ altura media; $\mathrm{n}=$ Número de dados observados; $p=$ Número de coeficientes do modelo.

O coeficiente de determinação ajustado $\left(\mathrm{R}_{\text {aj }}^{2}\right)$, o qual o coeficiente de determinação expressa a quantidade de variação total explicada pela regressão. Quanto mais próximo de 1 forem os valores dos coeficientes de determinação melhor será o ajuste da linha de regressão. Sendo assim, por este critério, seleciona-se o modelo matemático 
(equação 6) que apresentar o maior valor de $\mathrm{R}^{2}{ }_{\mathrm{aj}}$ (Schneider e Schneider, 2008).

$$
R^{2} \mathrm{aj}=\mathrm{R}-\left[\frac{K-1}{N-K}\right] \cdot\left(1-\mathrm{R}^{2}\right)
$$

Em que: $\mathrm{R}^{2} \mathrm{aj}=$ coeficiente de determinação ajustado; $\mathrm{R}^{2}=$ coeficiente de determinação; $\mathrm{K}=$ número de coeficientes da equação; $\mathrm{N}=$ número de observações.

A distribuição dos resíduos foi analisada plotando-se os desvios da regressão em gráfico. Observa-se a distribuição dos pontos e verifica-se se apresenta ou não tendências. Quando a distribuição dos resíduos da regressão apresenta-se homogênea, é considerado que a equação pertinente possui bom ajuste. A tendência de superestimar ou subestimar as estimativas pode ser observado na distribuição não uniforme dos dados ao longo da reta que correspondeu ao resíduo zero (Menon, 2005), a expressão (equação 7) elaborada pelo autor é:

$$
E_{i}=\left(\frac{Y_{i}-\widehat{Y}_{i}}{Y_{i}}\right) \cdot 100
$$

Em que: $\mathrm{E}_{\mathrm{i}}=$ resíduo da i-ésima observação; $Y_{\mathrm{i}}=$ valor real da árvore; $\widehat{Y}_{i}=$ valor estimado da árvore

Mesmo que todos os estimadores de ajuste sejam favoráveis para a seleção do melhor modelo, a análise gráfica de resíduos é decisiva, pois autoriza constatar se há ou não tendenciosidade na estimativa da variável dependente ao longo de toda linha de regressão, verificar se os resíduos são independentes, e se há homogeneidade de variância. Como a avaliação gráfica é visual, pode envolver alguma subjetividade, por isso é usada junto com as estatísticas citadas anteriormente (Miguel et al., 2010).

\section{RESULTADOS E DISCUSSÃO}

$\mathrm{Na}$ determinação da estatística descritiva (Tabela 2) observa-se que o CV\% para todos os clones e seminal apresentaram variação baixa para variável $\mathrm{Ht}(\mathrm{m})$ e de média para DAP $(\mathrm{cm})$ de acordo com a classificação proposta por Warrick \& Nielsen (1980), de baixa para CV $<12 \%$; média de $12 \%<\mathrm{CV}<60 \%$ e alta para CV $>60 \%$. Sendo a maior variação para DAP justificada pela maior amplitude de seus dados.

Tabela 2 - Estatística descritiva para DAP $(\mathrm{cm})$ e Ht $(\mathrm{m})$ para os híbridos Eucalyptus urophylla x Eucalyptus camaldulensis (VM 58), híbirdo de Eucalyptus urophylla $\mathrm{x}$ Eucalyptus grandis (GG100) e de sementes de Eucalyptus urophylla x Eucalyptus grandis aos 25 e 89 meses de idade em Aliança (TO).

\begin{tabular}{lccc}
\hline \multicolumn{1}{c}{ Estatísticas } & Híbrido & Híbrido & Seminal \\
& VM 58 & GG100 & GG100 \\
\hline DAP mínimo (cm) & 10,1 & 14,2 & 14 \\
DAP máximo (cm) & 24,6 & 25,4 & 21,9 \\
Média DAP $(\mathrm{cm})$ & 16,9 & 19,8 & 18,3 \\
DP DAP $(\mathrm{cm})$ & 1,27 & 2,11 & 2,24 \\
CV \% DAP & 9,11 & 12,67 & 15,31 \\
N de observações & 1634 & 487 & 839 \\
Ht Mínima (m) & 11 & 14 & 15 \\
Ht máxima (m) & 24 & 26,5 & 22 \\
Ht média (m) & 18,5 & 20,6 & 19,2 \\
DP Ht $(m)$ & 2,08 & 2,00 & 1,42 \\
CV \% Ht(m) & 10,82 & 10,1 & 7,4 \\
N de observações & 202 & 47 & 89 \\
\hline
\end{tabular}

O número de árvores cubadas por classe diamétrica (Tabela 3) foi correspondente à distribuição de frequência absoluta do povoamento por classe variando de 15 a $20 \%$. Esta metodologia também foi utilizada por Miranda et al. (2014) que ao avaliarem o efeito do método de amostragem da cubagem rigorosa na precisão de estimativas volumétricas em plantios clonais de Eucalyptus grandis W. Hill ex Maiden x Eucalyptus urophylla S. T. Blake, localizados na Bahia, com idade alterando de 4,8 a 5,4 anos, ajustaram o modelo de Schumacher-Hall a duas bases de dados, sendo a primeira com número fixo de árvores em todas as classes diamétricas e a segunda proporcional à distribuição diamétrica do povoamento. Os resultados demonstraram que a maior precisão foi obtida com a amostragem proporcional, o que permite redução do tempo e dos custos da operação de cubagem rigorosa. 
Tabela 3 - Distribuição das classes diamétricas de acordo com a altura total.

\begin{tabular}{cccccccc}
\hline DAP $(\mathrm{cm})$ & & \multicolumn{7}{c}{ Altura Total $(\mathrm{m})$} \\
\hline $12-16$ & $15-17$ & $17-19$ & $19-21$ & $21-23$ & $23-25$ & $25-27$ & Total \\
$16-20$ & 1 & 2 & 3 & 2 & 1 & 1 & 10 \\
$20-24$ & & 1 & 1 & 6 & 5 & 3 & 16 \\
\hline Total & 1 & 3 & 4 & 13 & 13 & 14 & 48 \\
\hline
\end{tabular}

A amostra piloto forneceu 14,94 árvores (com $\mathrm{CV}=38,86 \%$ para clone de Urocan), 15,34 árvores (com CV $=24,73 \%$ para clone de Urograndis) e de 13,12 árvores (com $\mathrm{CV}=$ de 48,97 para seminal) para assegurar o cálculo do volume individual foi realizado a cubagem do povoamento em 48 árvores para minimizar o erro, aos 7 anos de idade com fator de forma artificial calculado para cada árvore (Tabela 4).

Tabela 4 - Valores dos fatores de forma artificial individual obtidos pela cubagem rigorosa para o plantio comercial dos diferentes clones de Eucalyptus e via seminal aos 89 meses de idade.

\begin{tabular}{ccc|ccc|ccc}
\hline \multicolumn{3}{c}{ VM-58 } & \multicolumn{3}{c|}{ GG-100 } & \multicolumn{3}{c}{ Seminal } \\
\hline $\begin{array}{c}\text { Árvore } \\
\mathrm{N}^{0}\end{array}$ & $\begin{array}{c}\text { DAP } \\
(\mathrm{cm})\end{array}$ & $\begin{array}{c}\text { Fator de } \\
\text { forma }\end{array}$ & $\begin{array}{c}\text { Árvore } \\
\mathrm{N}^{0}\end{array}$ & $\begin{array}{c}\text { DAP } \\
(\mathrm{cm})\end{array}$ & Fator de forma & $\begin{array}{c}\text { Árvore } \\
\mathrm{N}^{0}\end{array}$ & $\begin{array}{c}\text { DAP } \\
(\mathrm{cm})\end{array}$ & Fator de forma \\
\hline 1 & 16,88 & 0,43 & 1 & 16,88 & 0,50 & 1 & 17,20 & 0,49 \\
2 & 18,15 & 0,44 & 2 & 13,69 & 0,47 & 2 & 11,46 & 0,40 \\
3 & 14,01 & 0,45 & 3 & 10,51 & 0,49 & 3 & 15,61 & 0,67 \\
4 & 13,38 & 0,46 & 4 & 12,74 & 0,36 & 4 & 16,56 & 0,48 \\
5 & 16,56 & 0,41 & 5 & 12,10 & 0,44 & 5 & 14,81 & 0,33 \\
6 & 18,15 & 0,45 & 6 & 16,88 & 0,42 & 6 & 19,11 & 0,64 \\
7 & 13,38 & 0,50 & 7 & 16,56 & 0,46 & 7 & 11,15 & 0,49 \\
8 & 16,56 & 0,47 & 8 & 15,92 & 0,45 & 8 & 13,06 & 0,52 \\
9 & 12,74 & 0,46 & 9 & 16,24 & 0,47 & 9 & 16,56 & 0,45 \\
10 & 18,15 & 0,51 & 10 & 11,15 & 0,45 & 10 & 11,46 & 0,39 \\
11 & 21,34 & 0,35 & 11 & 12,58 & 0,53 & 11 & 13,38 & 0,56 \\
12 & 16,24 & 0,47 & 12 & 14,55 & 0,47 & 12 & 16,88 & 0,52 \\
13 & 12,58 & 0,50 & 13 & 15,80 & 0,53 & 13 & 18,79 & 0,46 \\
14 & 14,68 & 0,44 & 14 & 18,15 & 0,54 & 14 & 19,11 & 0,44 \\
15 & 17,52 & 0,49 & 15 & 15,92 & 0,57 & 15 & 12,74 & 0,58 \\
16 & 18,15 & 0,50 & 16 & 12,26 & 0,49 & 16 & 13,69 & 0,42 \\
\hline
\end{tabular}

Nota-se que os fatores de forma diminuem com o aumento do diâmetro até permanecerem constantes nos maiores diâmetros. Para os Eucalyptus, os valores de fator de forma normalmente são próximos de 0,50 , ou seja, o volume real de madeira equivale a $50 \%$ do volume cilíndrico. Porém, os fatores de forma variam com o material genético, a idade e o sítio, podendo-se encontrar valores de 0,40 a 0,60 ou maiores. Amorim (2014) em estudos de fator de forma para Eucalyptus urophylla vs. Eucalyptus grandis encontraram valores variando de 0,5405 e 0,7658 tendo a mesma tendência dos valores encontrados neste estudo. Cipriani et al. (2013) também encontraram valores semelhantes a este estudo para o híbrido de Eucalyptus grandis $\mathrm{X}$ Eucalyptus urophylla aos 7,7 anos de idade com valor de 0,553 .

Verifica-se na tabela 5 que os clones não apresentaram diferença significativa a um nível de $95 \%$ de probabilidade de confiança para os parâmetros diâmetro à altura do peito (DAP), volume $\left(\mathrm{m}^{3}\right) \mathrm{e}$ Altura (m) com exceção do clone GG100 com o clone VM 58 para variável altura total $(\mathrm{m})$. 
Tabela 5 - Valores médios das variáveis biométricas DAP, volume individual, altura total, volume total dos diferentes clones de Eucalyptus e via seminal.

\begin{tabular}{llll}
\hline Variável & Clone VM 58 & Clone GG 100 & Seminal \\
\hline DAP $(\mathrm{cm})$ & $19,34^{\mathrm{ns}}$ & $18,53^{\mathrm{ns}}$ & $18,15^{\mathrm{ns}}$ \\
Volume $\left(\mathrm{m}^{3}\right)$ & $0,2555^{\mathrm{ns}}$ & $0,1725^{\mathrm{ns}}$ & $0,1911^{\mathrm{ns}}$ \\
Altura $(\mathrm{m})$ & $24,41^{\mathrm{ns}}$ & $22,3^{*}$ & $23,6^{\mathrm{ns}}$ \\
Volume total $\left(\mathrm{m}^{3} \cdot \mathrm{ha}^{-1}\right)$ & $255,562^{\mathrm{ns}}$ & $172,583^{\mathrm{ns}}$ & $191,147^{\mathrm{ns}}$ \\
\hline
\end{tabular}

Obs: Valores médios estimados pelo teste de Tukey com 95\% de probabilidade de confiança.

\section{Parâmetros das equações e estatísticas de precisão}

Os modelos que melhor se ajustaram aos dados foram Schumacher e Hall para VM 58 e Seminal de GG100 e Husch para GG 100 respectivamente
(Tabela 6), por apresentarem os melhores desempenhos, com maiores valores do coeficiente de determinação ajustado ( $\left.R^{2} a j\right)$ e menores valores do erro padrão da estimativa (Syx\%) e menores coeficientes de variação $(\mathrm{CV} \%)$.

Tabela 6 - Estimativas dos parâmetros estatísticos dos dez modelos matemáticos testados para descrever a relação volumétrica.

\begin{tabular}{|c|c|c|c|c|c|c|c|c|c|}
\hline \multicolumn{10}{|c|}{ Clone de VM-58 } \\
\hline Função & $\beta 0$ & $\beta 1$ & $\beta 2$ & $\beta 3$ & $\beta 4$ & $\beta 5$ & $\mathrm{R}^{2}$ ajust & SYX\% & $\mathrm{CV} \%$ \\
\hline Schumacher Hall & $-11,683$ & 1,4379 & 1,924584 & - & - & - & $\mathbf{0 , 8 8}$ & $\mathbf{8 , 8 3}$ & 5,00 \\
\hline Spur Log & $-8,9899$ & 0,8399 & - & - & - & - & 0,84 & 9,43 & 8,76 \\
\hline Meyer modificado & 0,6309 & $-0,0524$ & 0,000083 & 0,0033 & $-0,0000043$ & $-0,034$ & 0,85 & 15,21 & 9,22 \\
\hline Hohenald & $-0,0723$ & 0,0122 & 0,000231 & - & - & - & 0,74 & 19,87 & 8,56 \\
\hline Stoate & 0,0786 & $-0,0011$ & 0,000074 & $-0,0015$ & - & - & 0,87 & 14,29 & 9,19 \\
\hline Naslud & 0,1480 & $-0,0012$ & 0,000053 & 0,0000 & $-0,0004967$ & - & 0,86 & 14,29 & 9,22 \\
\hline Meyer & $-0,1118$ & 0,0509 & $-0,003459$ & $-0,0014$ & 0,0001513 & - & 0,86 & 14,56 & 9,22 \\
\hline Husch & $-6,6776$ & 1,7840 & - & - & - & - & 0,77 & 11,14 & 8,59 \\
\hline Brenac & $-3,3878$ & 0,8968 & $-13,039055$ & - & - & - & 0,76 & 11,51 & 8,31 \\
\hline Spurr & 0,0258 & 0,0000 & - & - & - & - & 0,83 & 15,81 & 35,80 \\
\hline \multicolumn{10}{|c|}{ Clone de GG-100 } \\
\hline Função & $\beta 0$ & $\beta 1$ & $\beta 2$ & $\beta 3$ & $\beta 4$ & $\beta 5$ & $R^{2}$ ajust & SYX\% & $\mathrm{CV} \%$ \\
\hline Schumacher Hall & $-11,183$ & 1,6900 & 1,48764 & - & - & - & 0,54 & 11,80 & 21,19 \\
\hline Spur Lc & $-9,8605$ & 0,9251 & - & - & - & - & 0,56 & 10,92 & 21,83 \\
\hline Meyer modificado & 54,302 & $-6,0359$ & 0,16632 & 0,2590289 & $-0,00709712$ & $-2,3351$ & 0,46 & 19,85 & 27,06 \\
\hline Hohenald & 0,2922 & $-0,0406$ & 0,00200 & - & - & - & 0,49 & 19,40 & 20,15 \\
\hline Stoate & 0,8448 & $-0,0030$ & 0,00014 & $-0,033605$ & - & - & 0,44 & 20,24 & 20,11 \\
\hline Naslud & 1316 & 0,0032 & $-0,00026$ & 0,0002424 & $-0,00253138$ & - & 0,49 & 19,37 & 21,38 \\
\hline Meyer & 0,2918 & $-0,0106$ & $-0,00026$ & $-0,001053$ & $8,3165 \mathrm{E}-05$ & - & 0,43 & 20,51 & 20,59 \\
\hline Husch & $-6,6776$ & 1,7840 & - & - & - & - & 0,77 & 11,42 & 34,89 \\
\hline Brenac & $-3,3878$ & 0,8968 & $-13,03905$ & - & - & - & 0,76 & 11,51 & 36,98 \\
\hline Spurr & $-0,0064$ & 0,0000 & - & - & - & - & 0,74 & 23,17 & 40,00 \\
\hline \multicolumn{10}{|c|}{ Seminal de GG-100 } \\
\hline Função & $\beta 0$ & $\beta 1$ & $\beta 2$ & $\beta 3$ & $\beta 4$ & $\beta 5$ & $\mathrm{R}^{2}$ ajust & SYX\% & $\mathrm{CV} \%$ \\
\hline Schumacher Hall & $-9,6712$ & 2,875982 & $-0,07626$ & - & - & - & 0,91 & 6,85 & 10,12 \\
\hline Spur Log & $-10,930$ & 1,046195 & - & - & - & - & 0,77 & 11,09 & 9,50 \\
\hline Meyer modificado & 4,4646 & $-0,56753$ & 0,01846 & 0,020575 & $-0,0006422$ & $-0,1634$ & 0,89 & 15,20 & 11,03 \\
\hline Hohenald & 0,3564 & $-0,051$ & 0,00236 & - & - & - & 0,89 & 15,31 & 10,89 \\
\hline Stoate & $-0,2669$ & 0,001654 & $-0,00003$ & 0,0071175 & - & - & 0,86 & 17,31 & 10,79 \\
\hline Naslud & $-0,1583$ & 0,000147 & 0,00010 & $-9,66 \mathrm{E}-05$ & 0,000939 & - & 0,87 & 16,59 & 10,90 \\
\hline Meyer & 0,5311 & $-0,10371$ & 0,00500 & 0,0013316 & $-0,000084$ & - & 0,89 & 15,10 & 10,99 \\
\hline Husch & $-6,6776$ & 1,784007 & - & - & - & - & 0,77 & 11,14 & 7,24 \\
\hline Brenac & $-3,3878$ & 0,896792 & $-13,03905$ & - & - & - & 0,76 & 11,51 & 7,01 \\
\hline Spurr & 0,1872 & 6,6E-06 & - & - & - & - & 0,69 & 15,05 & 7,66 \\
\hline
\end{tabular}


Em decorrência de gerar estimativas quase sempre não tendenciosas, devido às suas propriedades estatísticas, o modelo proposto por Schumacher e Hall é um dos mais utilizados na área florestal (Campos e Leite, 2009).

Cerdeira (2013), que mostrou que em um povoamento de Eucalyptus urograndis no Distrito Federal, o modelo de Schumacher e Hall (log) apresentou o maior valor de $\left(\mathrm{R}^{2} \mathrm{aj}\right)$, porém o seu (Syx \%) detém prioridade no ajuste e seleção de modelo, estatística essa que é superior ao outro modelo também testado o qual é o modelo de Takata. Miranda et al. (2014) em um plantio de Eucalyptus urograndis com 30 meses no Mato Grosso ao designarem o fator de forma e equações de volume para estimativa volumétrica de árvores, onde selecionaram a equação Schumacher-Hall (logarítmica) com $R^{2} a j$ 0,98 e CV\% de 3,63.

Azevedo et al. (2011) selecionaram o modelo que melhor se ajusta o de Schumacher-Hall logaritmizado com $\mathrm{R}^{2}$ aj de 0,981 e CV\% de 4,66 para volume total, que ao determinarem estimativas volumétricas para Eucalyptus aos 5 anos de idade sob regime de alto fuste e talhadia no sudoeste da Bahia. Azevedo et al. (2011), analisando equações volumétricas para um povoamento de Eucalyptus $s p$, com 35 anos de idade, sem espaçamento definido, no estado de Sergipe, o modelo que melhor se

Residuos Clone E. urophylla $\times$ E. camaldulensis (VM-58)

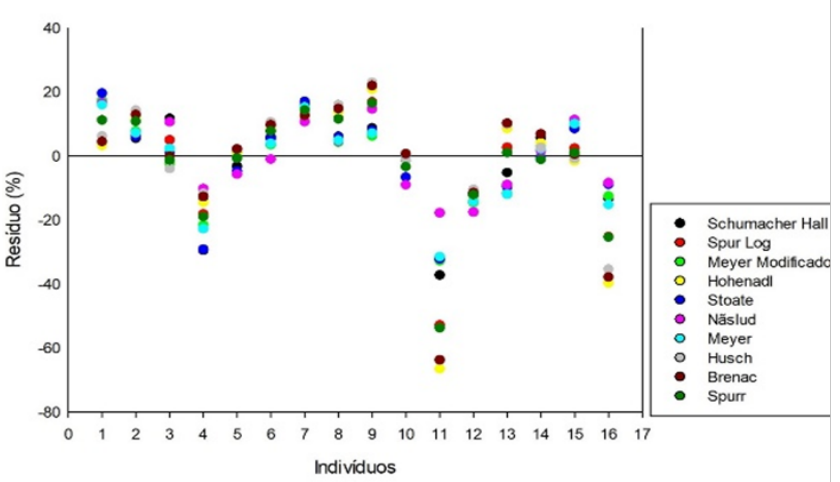

ajustou foi o modelo de Schumacher e Hall logaritimizado, mostrando o $\mathrm{R}^{2}$ ajustado de $87,45 \%$ e erro padrão da estimativa de $10,36 \%$ para volume com casca.

\section{Gráfico de distribuição de resíduos}

Nenhuma equação estimada deve ser usada antes de se proceder a uma análise gráfica de resíduos, mesmo que os índices de ajuste $\left(\mathrm{R}^{2}\right)$ e de precisão $(\mathrm{Syx} \%)$ sejam julgados satisfatórios (Draper e Smith, 2014). Essa análise, embora visual, é que realmente vai indicar se a estimativa é válida ao longo de toda a linha de regressão, se não há tendenciosidades em nenhum de seus segmentos e se a equação utilizada para as estimativas é realmente apropriada para tal fim. Por isso, foram feitos gráficos de análises de resíduos para todas as dezoito equações ajustadas para cada um dos clones e seminal. Após a seleção dos cinco melhores modelos, para estimativa de volumes com casca, procedeu-se à análise gráfica de resíduos para eles.

Todos os modelos foram ajustados para a análise gráfica de dispersão de resíduos (Figura 1), foram os modelos de Shumacher e Hall e Husch que apresentaram o melhor ajuste para o conjunto de dados entre as equações testadas, constatando os melhores modelos.
Residuos Clone de E. urophylla x E. grandis (GG-100)

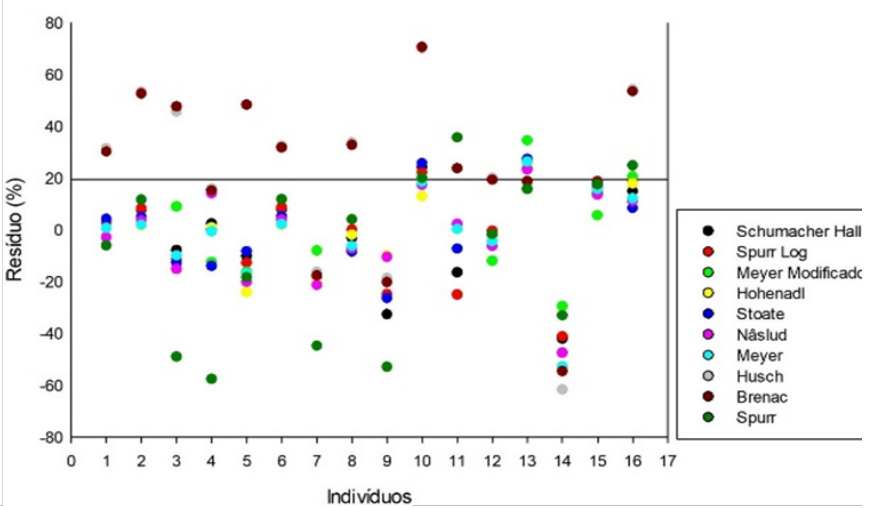

Resíduo Seminal de E. urophylla x E. grandis (GG-100)

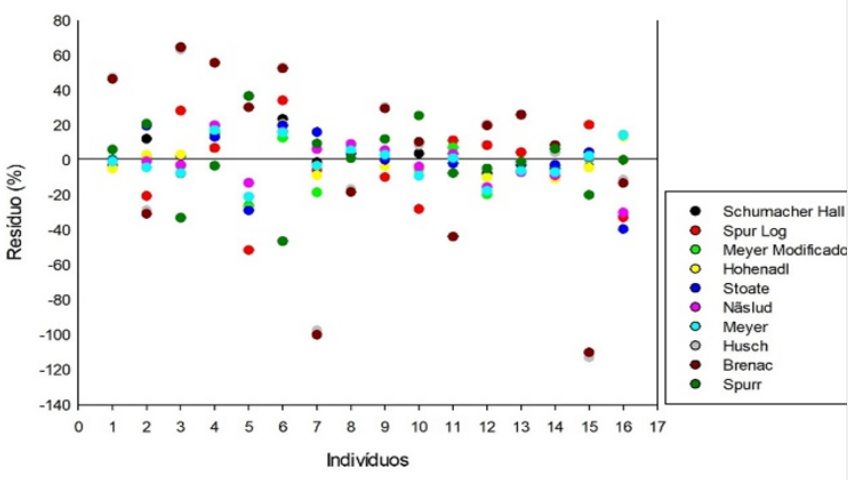

Figura 1 - Gráfico de distribuição de resíduos para VM-58, GG-100 e Seminal. 
A distribuição entre os valores observados e estimados neste estudo mostra que os modelos de Schumacher e Hall e Husch, apresentaram entre as equações testadas o melhor ajuste ao conjunto de dados do povoamento de VM-58, GG-100 e Seminal, respectivamente, evidenciando suas seleções como a melhor para cada um dos clones testados (Figura 2).
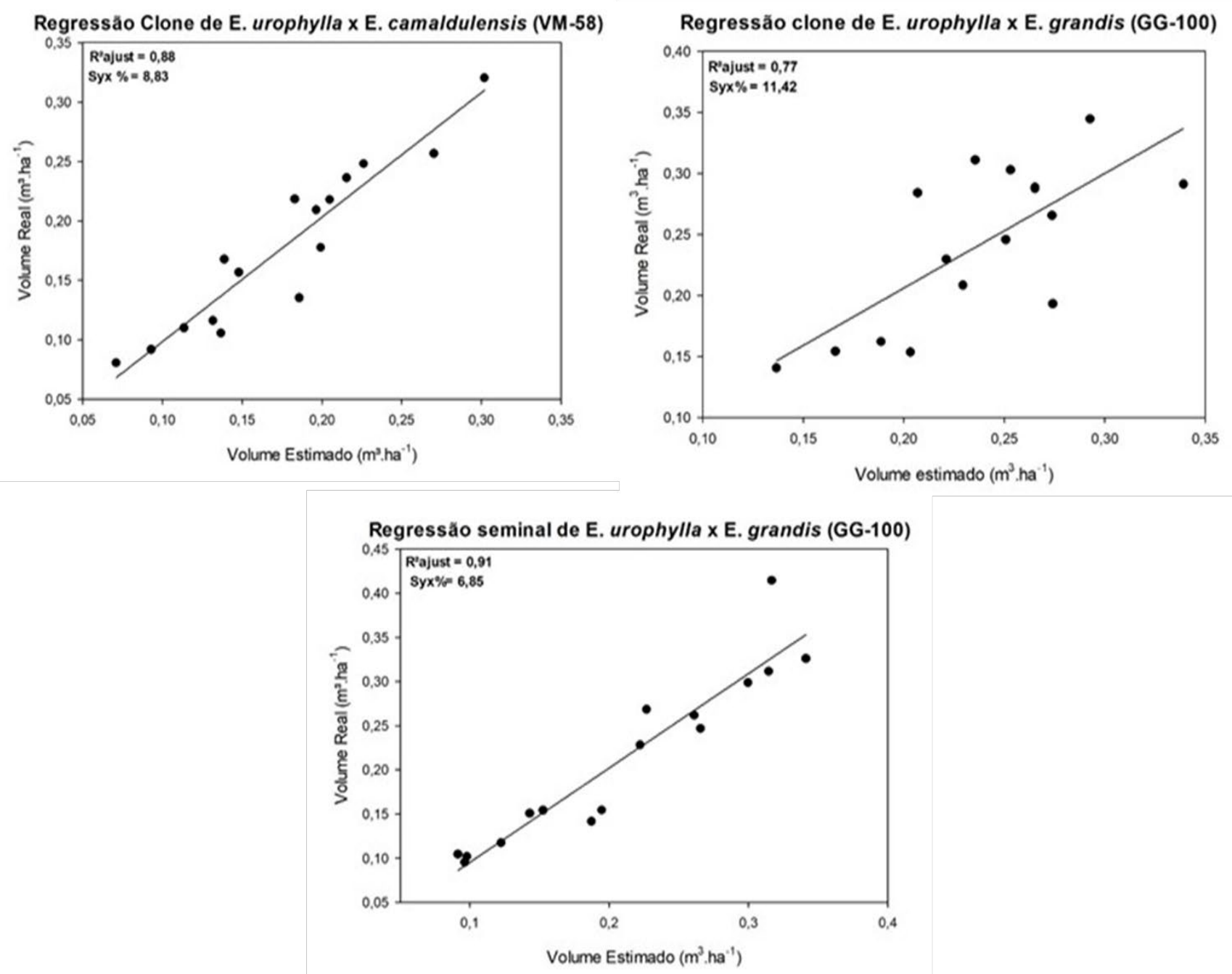

Figura 2 - Volume individual $\left(\mathrm{m}^{3}\right)$ estimado e observado por clone VM-58, GG-100 e Seminal de GG-100.

\section{CONCLUSÕES}

Não houve diferença significativa ao nível de 5\% pelo teste de Tukey entre os clones VM-58, GG100 e Seminal, nos parâmetros analisados de diâmetro a altura do peito (DAP) e volume. Entretanto para a média das alturas totais $(\mathrm{m}) \mathrm{o}$ clone GG-100 foi o que cresceu menos.

Os modelos matemáticos selecionados para descrever o volume total com casca foram o Schumacher e Hall e Husch confirmando estes serem os mais usados para Eucaliptos.

Os volumes totais foram GG $100=172,583$ $\mathrm{m}^{3} \cdot \mathrm{ha}^{-1}$, para VM $58=255,56 \mathrm{~m}^{3} \cdot \mathrm{ha}^{-1} \mathrm{e}$ para seminal $=191,147 \mathrm{~m}^{3} \cdot \mathrm{ha}^{-1}$.
Os fatores de forma encontrados estão na faixa esperada para eucaliptos $(0,40-0,60)$ podem ser utilizados para calcular o volume real de madeira em povoamentos de eucaliptos com características similares, bastando multiplicar o volume cilíndrico pelo respectivo fator de forma.

\section{REFERÊNCIAS BIBLIOGRÁFICAS}

Amorim DM. Fator de forma artificial para o híbrido Eucalyptus urophylla vs Eucalyptus grandis na Amazônia Oriental. 2014. Monografia (Graduação em Engenharia Florestal) Universidade Federal do Pará, Altamira, 2014.

Azevedo TL, Mello AAD, Ferreira RA, Sanquetta CR, Nakajima NY. Equações hipsométricas e volumétricas para um 
povoamento de Eucalyptus sp. localizado na FLONA do Ibura, Sergipe. Revista Brasileira de Ciências Agrárias, 6 (1): 105-122p. 2011. https://doi.org/DOI:10.5039/agraria.v6ila861

Batista JLF, Couto HTZ, Marquesini M. Desempenho de modelos de relações hipsométricas: estudo em três tipos de floresta. Scientia Forestalis, 1 (60): 143-163p.2001.

Campos JCC, Leite HG. Mensuração florestal: perguntas e respostas. Minas Gerais: Viçosa. 94p.2009.

Carvalho M, Rosa M, Lima G, Koehler, HS, Nappo ME. Comparação dos métodos de cubagem na determinação do volume de árvores de Eucalyptus urophylla x Eucalyptus grandis. Atualidades em Mensuração Florestal. Paraná: Curitiba. 50-54p.2014

Cerdeira ALN. Modelos para quantificação do volume de diferentes sortimentos em plantio de Eucalyptus urophylla $\mathrm{x}$ Eucalyptus grandis. Monografia, Graduação Engenharia Florestal, Universidade de Brasília. 66p. 2013.

Cipriani HN, Vieira AH, Godinho VPC. Crescimento inicial de clones de eucalipto em Vilhena, RO. Porto Velho: EMPRABA, 2013. (Comunicado Técnico, 388).

Draper NR, Smith H. Applied regression analysis. $3^{\text {a }}$ Edition. Canada: Wiley \& Sons. 326p. 2014.

Finger CAG. Fundamentos de biometria florestal. Santa Maria: Universidade Federal de Santa Maria. 269p.1992.

IBÁ. Indústria Brasileira de Árvores. São Paulo. 100p. 2017.

Machado SA, Urbano E, Conceição MB. Comparação de métodos de estimativa de volume para Pinus oocarpa em diferentes idades e diferentes regimes de desbastes. Pesquisa Florestal Brasileira, v.1, n.50, 81-98p.2005.

Mello JM. Geoestatística aplicada ao inventário florestal. Tese de Doutorado, Universidade de São Paulo, Piracicaba. 122, p.2004.

Menon MU. Ajuste dos modelos de Kozak e do sistema Murkhart e Cao para plantações de Pinus oocarpa. Revista Ambiência, 1: 197-205p.2005.

Miguel EP, Canzi LF, Rufino RF, Santos GD. Ajuste de modelo volumétrico e desenvolvimento de fator de forma para plantios de Eucalyptus grandis localizados no município de Rio Verde - GO. Enciclopédia Biosfera, 6 (11): 1-13p. 2010.

Miguel E P, Leal FA, Ono HA, Leal UAS. Modelagem na predição do volume individual em plantio de Eucalyptus urograndis. Revista Brasileira de Biometria, 32 (4): 584598, p.2014.

Miranda RO, Ebling AA, David HC, Fiorentin LD, Soares ID. Efeito do método de amostragem da cubagem rigorosa na precisão de estimativas volumétricas. In: Atualidades em Mensuração Florestal. Curitiba: Paraná Brasil. 67-70p. 2014.

Santana OA, Encinas JI. Equações volumétricas para uma plantação de Eucalyptus urophylla destinada a produção de lenha. In: Simpósio latino-americano sobre manejo florestal. Santa Maria: UFSM/PPGEF. 107-111p. 2004.

Schneider PR, Schneider PS. Introdução ao manejo florestal. 2 Edition. Santa Maria: FACOS -UFSM. 566p. 2008.
SEPLAN - Secretária do Planejamento. Diretoria de Zoneamento Ecológico- Economico Base de dados geográfico do TO. Palmas: Tocantins.2012.

Silva JAA, Paula NF. Princípios básicos de dendrometria. 1 Edition. Recife: Universidade Federal Rural do Pernambuco. 191p. 1979.

Thomas C, Andrade CM, Schneider PR, Finger CAG. Comparação de equações volumétricas ajustadas com dados de cubagem e análise de tronco. Ciência Florestal, 16 (3): 319-327p. 2006.

Viera M, Schumacher MV, Trüby P, Araújo EF. Biomassa e nutrientes em um povoamento de Eucalyptus urophylla $\mathrm{x}$ Eucalyptus globulus, em Eldorado do Sul-RS. Revista Ecologia e Nutrição Florestal-ENFLO, 1(1): 1-13p.2014.

Vital MHF. Impacto ambiental de florestas de eucalipto. Revista do BNDES, Rio de Janeiro, 14: 235-276p. 2007. 